\title{
Synthesis of 2-cyclopropyl-3-(5-aryl-1H-pyrazol- 3-yl)-1,8-naphthyridine
}

\author{
Mallaiah Bucha ${ }^{1}$, Laxminarayana Eppakayala ${ }^{1}$, Thirumala Chary Maringanti ${ }^{2 *}$, Giri Tharikoppula ${ }^{1}$ \\ and Shashikala Kethireddy ${ }^{2}$
}

\begin{abstract}
Background: 1,8-Naphthyridine derivatives have attracted considerable attention because the 1,8-naphthyridine skeleton is present in many compounds that have been isolated from natural substances, with various biological activities.

Findings: $\mathrm{N}, \mathrm{N}$-dimethoxy-N-methyl-1,8-naphthyridine-3-carboxamide (1) on reaction with Grignard reagent forms 2-methoxy-1,8-naphthyridine-3-carbaldehyde (2). Compound 2 on reaction with different aromatic aldehydes provided 1-(2-cyclopropyl-1,8-naphthyridin-3-yl)-3-arylprop-2-en-1-ones (3a-e) and these compounds on cyclisation with hydrazine hydrate 99\% yielded 2-cyclopropyl-3-(5-aryl-1H-pyrazol-3-yl)-1,8-naphthyridines (4-a-e).

Synthesis of the target compounds involved the formation of 4a-e. It was accomplished using Grignard reaction, condensation reaction, and cyclisation reactions. All the synthesized compounds were readily soluble in DMSO. Spectral data of the synthesized compounds were in full agreement with the proposed structures.
\end{abstract}

Conclusions: In conclusion, we have developed a simple and an efficient Synthesis of 2-cyclopropyl-3-(5-aryl-1H-pyrazol3-yl)-1,8-naphthyridine.

Keywords: Grignard reagent; Cyclisation; 1,8-naphthyridine

\section{Findings}

\section{Background}

Among the wide variety of heterocycles that have been explored for developing pharmaceutically important molecules, such as chalcones, pyrazolines and amino pyrimidines have played an important role in medicinal chemistry. The presence of reactive $\alpha, \beta$-unsaturated carbonyl function in chalcones is found to be responsible for their antibacterial and antifungal activity. Nitrogen containing heterocyclic compounds find extensive pharmaceutical applications and possess biologically activity. Many of the naphthyridines have shown bacterial, fungicidal and carcinogenic activity [1-3]. As a step in this direction and in continuation of our work on 1, 8-naphthyridines [4-7], synthesis of the title compounds was carried out. The general synthetic procedures used in the preparation of these compounds involved the cyclisation of Schiff's bases [8-12].

\footnotetext{
* Correspondence: mtcharya@yahoo.com

2Jawaharlal Nehru Technological University Hyderabad College of Engineering, Nachupally, Karimnagar 505501 Telangana, India Full list of author information is available at the end of the article
}

The structure of the compounds were confirmed on the basis of their spectral ( ${ }^{1} \mathrm{H}$ NMR and mass) data. The synthetic approach is outlined in Scheme 1.

\section{Methods}

A simple conventional method is followed to prepare all the title compounds.

\section{Results and discussion}

Compound (1), when reacted with Grignard reagent and gave 2-methoxy-1,8-naphthyridine-3-carbaldehyde (2) which was reacted with different aldehydes to form arylidene derivatives (3a-e), which on treatment with hydrazine hydrate afforded compound (4a-e).

\section{Experimental}

Melting points were determined in open capillaries and are uncorrected. ${ }^{1} \mathrm{H}$ NMR spectrum is taken on a Varian $500 \mathrm{MHz}$ instrument with TMS as an internal standard. The chemical shifts are expressed $\delta \mathrm{ppm}$, and the solvent used is DMSO- $\mathrm{d}_{6}$. Mass spectrum is taken on HewlettPackard mass spectrometer operating at $70 \mathrm{eV}$. All the compounds are recrystalised from ethanol.

\section{它}

(c) 2014 Tharikoppula et al; licensee Springer. This is an Open Access article distributed under the terms of the Creative Commons Attribution License (http://creativecommons.org/licenses/by/4.0), which permits unrestricted use, distribution, and reproduction in any medium, provided the original work is properly credited. 


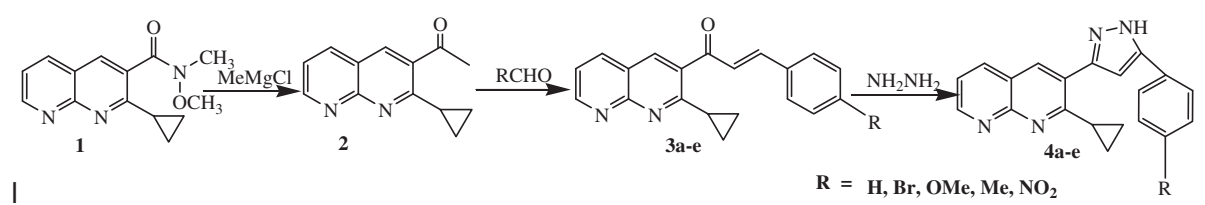

Scheme 1 Synthesis of 1,8-naphthyridines.

\section{1-(2-cyclopropyl-1,8-naphthyridin-3-yl)ethanone (2)}

To a solution of 2-cyclopropyl- $N$-methoxy- $N$-methyl1,8-naphthyridine-3-carboxamide (1) (1.01 $\mathrm{mmol})$ in THF $(10 \mathrm{~mL}), \mathrm{MeMgCl}(3.03 \mathrm{mmol})$ was added drop wise at $-30^{\circ} \mathrm{C}$ and stirred for about $2 \mathrm{~h}$ at $-30^{\circ} \mathrm{C}$. The resulting solution is quenched with a saturated $\mathrm{NH}_{4} \mathrm{Cl}$ solution $(30 \mathrm{~mL})$, filtered, extracted with EtOAc $(3 \times$ $30 \mathrm{~mL}$ ) and dried over $\mathrm{Na}_{2} \mathrm{SO}_{4}$. The resulting crude compound was purified by a column chromatography by eluting $20 \%$ to $40 \%$ ethyl acetate in hexane to obtain the light yellow colour solid.

${ }^{1} \mathrm{H}$ NMR (DMSO- $\left.d_{6}, 500 \mathrm{MHz}\right): \delta$ 1.06-1.21 (m, 4H, 2CH2), $2.24\left(\mathrm{~s}, 3 \mathrm{H}, \mathrm{CH}_{3}\right), 2.57(\mathrm{~m}, 1 \mathrm{H}, \mathrm{CH}), 7.57(\mathrm{t}, 1 \mathrm{H}, J=$ $12 \mathrm{~Hz} \mathrm{CH}), 8.31$ (s, $1 \mathrm{H}, J=12 \mathrm{~Hz} \mathrm{CH}), 8.42$, (d, $1 \mathrm{H}, \mathrm{CH})$, $9.02(\mathrm{~d}, 1 \mathrm{H}, J=10 \mathrm{~Hz} \mathrm{CH})$. Mass $[\mathrm{M}+1]$ peak at $m / z 213$.

\section{1-(2-cyclopropyl-1,8-naphthyridin-3-yl)-3-phenylprop-2-en-1-} one (3a)

To a solution of 1-(2-cyclopropyl-1,8-naphthyridin-3-yl) ethanone (2) (0.53 mmol) in DMF, $\mathrm{NaOH}(0.79 \mathrm{mmol})$ and aldehyde $(0.63 \mathrm{mmol})$ were added at $0{ }^{\circ} \mathrm{C}$ and stirred for about $1 \mathrm{~h}$. Then water was added and extracted with DCM $(2 \times 15 \mathrm{~mL})$, dried over $\mathrm{Na}_{2} \mathrm{SO}_{4}$ and evaporated under reduced pressure. The resulting crude compound was purified by a column chromatography by eluting $20 \%$ to $40 \%$ ethyl acetate in hexane to obtain the light yellow colour solid.

${ }^{1} \mathrm{H}$ NMR (DMSO- $\left.d_{6}, 500 \mathrm{MHz}\right): \delta$ 1.12-1.28 (m, 4H, $\left.2 \mathrm{CH}_{2}\right), 2.61(\mathrm{~m}, 1 \mathrm{H}, \mathrm{CH}), 7.62(\mathrm{~m}, 2 \mathrm{H}, J=14 \mathrm{~Hz} 2 \mathrm{CH}), 7.81$ $(\mathrm{dd}, 2 \mathrm{H}, J=18 \mathrm{~Hz} 2 \mathrm{CH}), 8.23,(\mathrm{~m}, 3 \mathrm{H}, 3 \mathrm{CH}), 8.51-8.61$ (m, $4 \mathrm{H}, J=18 \mathrm{~Hz} 4 \mathrm{CH}), 9.12(\mathrm{~s}, 1 \mathrm{H}, \mathrm{CH})$. Mass $[\mathrm{M}+1]$ peak at $m / z 301$.

Other compounds in the series were prepared similarly and their characterization data are recorded below.

\section{3-(4-bromophenyl)-1-(2-cyclopropyl-1,8-naphthyridin-3-yl)} prop-2-en-1-one (3b)

${ }^{1} \mathrm{H}$ NMR (DMSO- $\left.d_{6}, 500 \mathrm{MHz}\right): \delta$ 1.14-1.29 (m, 4H, $\left.2 \mathrm{CH}_{2}\right), 2.52,(\mathrm{~m}, 1 \mathrm{H}, \mathrm{CH}), 7.62(\mathrm{~m}, 4 \mathrm{H}, 4 \mathrm{CH}), 7.71(\mathrm{~d}$, $2 \mathrm{H}, J=12 \mathrm{~Hz} 2 \mathrm{CH}), 8.23,(\mathrm{~d}, 1 \mathrm{H}, 1 \mathrm{CH}), 8.69(\mathrm{~s}, 1 \mathrm{H}$, $1 \mathrm{CH}), 9.22(\mathrm{~s}, 1 \mathrm{H}, \mathrm{CH})$.Mass $[\mathrm{M}+1] \mathrm{m} / z 380$.

\section{1-(2-cyclopropyl-1,8-naphthyridin-3-yl)-3-(4-methoxyphenyl) prop-2-en-1-one (3c)}

${ }^{1} \mathrm{H}$ NMR (DMSO- $\left.d_{6}, 500 \mathrm{MHz}\right): \delta$ 1.16-1.27 (m, 4H, 2CH2), 2.48 (m, 1H, CH), $7.62(\mathrm{~m}, 4 \mathrm{H}, 4 \mathrm{CH}), 7.69$ (d,
$2 \mathrm{H}, J=12 \mathrm{~Hz} 2 \mathrm{CH}$ ), 8.33 (d, $1 \mathrm{H}, J=12 \mathrm{~Hz}, 1 \mathrm{CH}), 8.71(\mathrm{~s}$, $1 \mathrm{H}, 1 \mathrm{CH}), 9.24(\mathrm{~s}, 1 \mathrm{H}, \mathrm{CH})$. Mass $[\mathrm{M}+1]$ peak at $m / z 331$.

1-(2-cyclopropyl-1,8-naphthyridin-3-yl)-3-p-tolylprop-2-en-1one $(3 d)$

${ }^{1} \mathrm{H}$ NMR (DMSO- $\left.d_{6}, 500 \mathrm{MHz}\right): \delta$ 1.19-1.28 (m, $4 \mathrm{H}$, $\left.2 \mathrm{CH}_{2}\right), 2.53(\mathrm{~m}, 1 \mathrm{H}, \mathrm{CH}), 7.63(\mathrm{~m}, 4 \mathrm{H}, 4 \mathrm{CH}), 7.72(\mathrm{~d}, 2 \mathrm{H}$, $J=14 \mathrm{~Hz} 2 \mathrm{CH}), 8.42(\mathrm{~d}, 1 \mathrm{H}, J=14 \mathrm{~Hz}, 1 \mathrm{CH}), 8.78(\mathrm{~s}, 1 \mathrm{H}$, $1 \mathrm{CH}), 9.28(\mathrm{~s}, 1 \mathrm{H}, \mathrm{CH})$.Mass $[\mathrm{M}+1]$ peak at $m / z 315$.

\section{1-(2-cyclopropyl-1,8-naphthyridin-3-yl)-3-(4-nitrophenyl)} prop-2-en-1-one (3e)

${ }^{1} \mathrm{H}$ NMR (DMSO- $\left.d_{6}, 500 \mathrm{MHz}\right): \delta$ 1.23-1.31 (m, 4H, $\left.2 \mathrm{CH}_{2}\right), 2.62(\mathrm{~m}, 1 \mathrm{H}, \mathrm{CH}), 7.73(\mathrm{~m}, 4 \mathrm{H}, 4 \mathrm{CH}), 7.81(\mathrm{~d}, 2 \mathrm{H}$, $J=18 \mathrm{~Hz} 2 \mathrm{CH}), 8.56(\mathrm{~d}, 1 \mathrm{H}, J=16 \mathrm{~Hz}, 1 \mathrm{CH}), 8.64(\mathrm{~s}, 1 \mathrm{H}$, $1 \mathrm{CH}), 9.32(\mathrm{~s}, 1 \mathrm{H}, \mathrm{CH})$.Mass $[\mathrm{M}+1]$ peak at $m / z 346$.

\section{2-cyclopropyl-3-(5-phenyl-1H-pyrazol-3-yl)-1,8-} naphthyridine (4a)

To a solution of 1-(2-cyclopropyl-1,8-naphthyridin-3-yl)3-phenylprop-2-en-1-one (3a) $(0.31 \mathrm{mmol})$ in ethanol $(5 \mathrm{~mL})$, hydrazine hydrate $99 \%(2 \mathrm{~mL})$ was added. The resulting solution was refluxed for about $12 \mathrm{~h}$. After completion of a starting material, the ethanol was evaporated completely under a reduced pressure then the title compound was recrystalised from diethyl ether to obtain the brown colour solid.

${ }^{1} \mathrm{H}$ NMR (DMSO- $\left.d_{6}, 500 \mathrm{MHz}\right): \delta 1.16\left(\mathrm{~m}, 2 \mathrm{H}, \mathrm{CH}_{2}\right)$, $1.22\left(\mathrm{~m}, 2 \mathrm{H}, \mathrm{CH}_{2}\right), 2.21(\mathrm{~m}, 1 \mathrm{H}, \mathrm{CH}), 5.21(\mathrm{~s}, 1 \mathrm{H}, J=12 \mathrm{~Hz}$ $\mathrm{CH}), 5.41(\mathrm{~s}, 1 \mathrm{H}, J=12 \mathrm{~Hz} \mathrm{CH}), 6.31(\mathrm{~m}, 1 \mathrm{H}, J=12 \mathrm{~Hz} \mathrm{CH})$, 6.59, (m, 2H, 2CH), $7.19(\mathrm{~d}, 1 \mathrm{H}, J=10 \mathrm{~Hz} \mathrm{CH}), 7.69(\mathrm{~m}$, $4 \mathrm{H}, 4 \mathrm{CH}), 8.42$ (m, 2H, 2CH), $9.11(\mathrm{~s}, 1 \mathrm{H}, \mathrm{CH}), 10.81$ (s, $1 \mathrm{H}, \mathrm{NH})$. Mass $[\mathrm{M}+1]$ peak at $m / z 313$.

Other compounds in the series were prepared similarly and their characterization data are recorded below.

\section{3-(5-(4-bromophenyl)-1H-pyrazol-3-yl)-2-cyclopropyl-1,8-} naphthyridine (4b)

${ }^{1} \mathrm{H}$ NMR (DMSO- $\left.d_{6}, 500 \mathrm{MHz}\right): \delta 1.21\left(\mathrm{~m}, 2 \mathrm{H}, \mathrm{CH}_{2}\right)$, $1.28\left(\mathrm{~m}, 2 \mathrm{H}, \mathrm{CH}_{2}\right), 2.37(\mathrm{~m}, 1 \mathrm{H}, \mathrm{CH}), 6.21(\mathrm{~s}, 1 \mathrm{H}, J=$ $12 \mathrm{~Hz} \mathrm{CH}), 7.32(\mathrm{~s}, 1 \mathrm{H}, \mathrm{CH}), 7.41(\mathrm{~m}, 4 \mathrm{H}, J=12 \mathrm{~Hz} 4 \mathrm{CH})$, 8.19 (m, 2H, 2CH), 8.39 (d, 1H, CH), 10.69 (s, 1H, NH). Mass $[M+2]$ peak at $m / z 392$. 
2-cyclopropyl-3-(5-(4-methoxyphenyl)-1H-pyrazol-3-yl)-1,8naphthyridine (4c)

${ }^{1} \mathrm{H}$ NMR (DMSO- $\left.d_{6}, 500 \mathrm{MHz}\right): \delta 1.24\left(\mathrm{~m}, 2 \mathrm{H}, \mathrm{CH}_{2}\right)$, $1.29\left(\mathrm{~m}, 2 \mathrm{H}, \mathrm{CH}_{2}\right), 2.28(\mathrm{~m}, 1 \mathrm{H}, \mathrm{CH}), 4.12\left(\mathrm{~s}, 3 \mathrm{H}, \mathrm{CH}_{3}\right)$, $6.23(\mathrm{~s}, 1 \mathrm{H}, \mathrm{CH}), 7.35(\mathrm{~s}, 1 \mathrm{H}, \mathrm{CH}), 7.29(\mathrm{~m}, 4 \mathrm{H}, J=12 \mathrm{~Hz}$ $4 \mathrm{CH}), 8.03,(\mathrm{~m}, 2 \mathrm{H}, 2 \mathrm{CH}), 8.42(\mathrm{~d}, 1 \mathrm{H}, \mathrm{CH}), 10.46(\mathrm{~s}, 1 \mathrm{H}$, $\mathrm{NH})$. Mass $[\mathrm{M}+1]$ peak at $m / z 343$.

\section{2-cyclopropyl-3-(5-p-tolyl-1H-pyrazol-3-yl)-1,8-naphthyridine} (4d)

${ }^{1} \mathrm{H}$ NMR (DMSO- $\left.d_{6}, 500 \mathrm{MHz}\right): \delta 1.18\left(\mathrm{~m}, 2 \mathrm{H}, \mathrm{CH}_{2}\right)$, $1.31\left(\mathrm{~m}, 2 \mathrm{H}, \mathrm{CH}_{2}\right), 2.20(\mathrm{~m}, 1 \mathrm{H}, \mathrm{CH}), 2.39\left(\mathrm{~s}, 3 \mathrm{H}, \mathrm{CH}_{3}\right)$, 6.28 (s, 1H, CH), 7.25 (s, 1H, CH), $7.39(\mathrm{~m}, 4 \mathrm{H}, 4 \mathrm{CH}), 8.23$, (m, $2 \mathrm{H}, 2 \mathrm{CH}), 8.44(\mathrm{~d}, 1 \mathrm{H}, J=12 \mathrm{~Hz}, \mathrm{CH}), 10.59(\mathrm{~s}, 1 \mathrm{H}$, $\mathrm{NH})$. Mass $[\mathrm{M}+1]$ peak at $m / z 327$.

\section{2-cyclopropyl-3-(5-(4-nitrophenyl)-1H-pyrazol-3-yl)-1,8- naphthyridine (4e)}

${ }^{1} \mathrm{H}$ NMR (DMSO- $\left.d_{6}, 500 \mathrm{MHz}\right): \delta 1.18\left(\mathrm{~m}, 2 \mathrm{H}, \mathrm{CH}_{2}\right), 1.31$ (m, 2H, $\mathrm{CH}_{2}$ ), 2.20 (m, 1H, CH), 6.18 (s, 1H, CH), 7.15 (s, $1 \mathrm{H}, \mathrm{CH}), 7.27$ (m, 4H, 4CH), 8.12, (m, 2H, 2CH), 8.24 (d, $1 \mathrm{H}, J=12 \mathrm{~Hz}, \mathrm{CH}), 11.19(\mathrm{~s}, 1 \mathrm{H}, \mathrm{NH})$. Mass $[\mathrm{M}+1]$ peak at $m / z 358$.

\section{Conclusions}

We have presented simple, cost effective, and practical method for preparation of some 1,8-naphthyridines. This methodology provides an efficient alternative to existing methods for the synthesis of 2-cyclopropyl-3-(5aryl-1H-pyrazol-3-yl)-1,8-naphthyridine. Here in step-1, we observed the formation of Grignard product which on condensation of aldehydes Schiffs base is formed. Further reaction Schiffs base with hydrazine we obtained the pyrazoles.

\section{Competing interests}

The authors declare that they have no competing interests.

\section{Author details}

${ }^{1}$ Mahatma Gandhi Institute of Technology, Gandipet, Hyderabad 50075

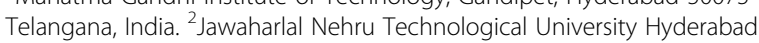
College of Engineering, Nachupally, Karimnagar 505501 Telangana, India.

Received: 21 August 2014 Accepted: 22 September 2014

Published online: 21 December 2014

\section{References}

1. Nezval J, Halocka J (1967) The enhancing effect on the antibacterial activity of nalidixix acid against Pseudomonas aeruginosa. Experientia 23:1043

2. Egawa H, Miyamido A, Nishimra Y, Okada H, Uno H, Matsumato J (1984) Pyridonecarboxylic acids as antibacterial agents. J Med Chem 27:1543

3. Suzuki N, Dohmori R (1979) The chemistry of heterocyclic compounds: the naphthyridines. Chem Pharma Bull 27:410

4. Shiva Shankar S, Laxminarayana E, Haripriya V, Thirumala Chary M (2011) Synthesis of 5-(2-methoxy-1,8-naphthyridin-3-yl)-4-aryl-4H-1,2,4-triazole-3thiols and 5-(2-methoxy-1,8-naphthyridin-3-yl)-n-aryl-1,3,4-thiadiazol-2amines. Indian J Heterocycl Chem 21:101

5. Narender A, Thirumala Chary M, Laxminarayana E, Haripriya V (2013) Synthesis and antimicrobial activity of 2-cyclopropyl [1,8]naphthyridine-3carboxylic acid (4-phenyl-2-thioxo-thiazol-3-yl)-amides, [1,3,5]triazine, [1,3,4] thiadiazole-2-thiol, [1,2,4]triazole-3-thiol and coumarine derivatives. Indian J Chem 52B:440
6. Mallaiah B, Shiva Shankar S, Laxminarayana E, Thirumala Chary M (2013) Synthesis of 2-(5-(2-methoxy-1,8-naphthyridin-3-yl)-1,3,4-oxadiazol/triazol-2ylthio)-1-(4-alkylbenzofuran-2-yl)ethanones and 2-methoxy-3-(5-(4-alkylbenzofuran-2-yl)-7aH-thiazolo[2,3-b][1,3,4]oxadiazol/triazol-2-yl)-1,8-naphthyridines by conventional and eco-friendly methods and evaluation of their antibacterial activity. Indian J Heterocycl Chem 22(2):333

7. Shiva Shankar E, Laxminarayana M, Thirumala C (2013) Synthesis of 5-(2methoxy-1,8-naphthyridin-3-yl)-1,3,4-oxadiazole-2(3H)-thione and 1-(2-methoxy-1,8-naphthyridin-3-acetyl)-4-arylthiosemicarbazides. Indian J Heterocycl Chem 23(2):139

8. Vadim A, Soloshonok CC, Yamada T, Hisanori U, Ohfune Y, Hruby VJ (2005) Michael addition reactions between chiral equivalents of a nucleophilic glycine and (S)- or (R)-3-[(E)-Enoyl]-4-phenyl-1,3-oxazolidin-2-ones as a general method for efficient preparation of $\beta$-substituted pyroglutamic acids: case of topographically controlled stereoselectivity. J Am Chem Soc 43:127

9. Reddy KR, Kumar NS (2006) Cellulose-supported copper(0) catalyst for Aza-Michael addition. Synlett 6:224

10. Wu F, Li L, Hong R, Deng L (2006) Construction of quaternary stereocenters via efficient and practical conjugate additions to a, $\beta$-unsaturated ketones with a chiral organic catalyst. Angew Chem Int 45:947

11. Bensa D, Constantieux T, Rodriguez J (2004) A new efficient and commercially available user-friendly and recyclable heterogeneous organocatalyst for the Michael addition of 1,3-dicarbonyl compounds. Synthesis 06:923

12. Ooi T, Ohara D, Fukumoto K, Maruoka K (2005) Importance of chiral phasetransfer catalysts with dual functions in obtaining high enantioselectivity in the Michael reaction of malonates and chalcone derivatives. Org Lett 7:3195

\section{Submit your manuscript to a SpringerOpen ${ }^{\circ}$ journal and benefit from:}

- Convenient online submission

- Rigorous peer review

- Immediate publication on acceptance

- Open access: articles freely available online

- High visibility within the field

- Retaining the copyright to your article

Submit your next manuscript at $>$ springeropen.com 